\title{
Validation of a New Procedure for Impedance Eduction in Flow
}

\author{
W. R. Watson* and M.G. Jones ${ }^{\dagger}$ \\ NASA Langley Research Center, Hampton, Virginia 23681-2199, USA
}

\begin{abstract}
A new impedance eduction procedure is validated by comparing the educed impedance spectrum to that of an older but well-tested eduction procedure. The older procedure requires the installation of a microphone array in the liner test section but the new procedure removes this requirement. A 12.7-mm stainless steel plate and a conventional liner consisting of a perforated plate bonded to a honeycomb core are tested. Test data is acquired from a grazing flow, impedance tube facility for a range of source frequencies and mean flow Mach numbers for which only plane waves are cut on. For the stainless steel plate, the educed admittance spectrum using the new procedure shows an improvement over that of the old procedure. This improvement shows up primarily in the educed conductance spectrum. Both eduction procedures show discrepancies in educed admittance in the mid-frequency range. Indications are that this discrepancy is triggered by an inconsistency between the measured eduction data (that contains boundary layer effects) and the two eduction models (for which the boundary layer is neglected). For the conventional liner, both eduction procedures are in very good agreement with each other. Small discrepancies occur for one or two frequencies in the mid-frequency range and for frequencies beyond the cut on frequency of higher-order duct modes. This discrepancy in the midfrequency range occurs because an automated optimizer is used to educe the impedance and the objective function used by the optimizer is extremely flat and therefore sensitive to initial starting values. The discrepancies at frequencies beyond the cut on frequency of higher order duct modes are due to the assumption of only plane waves in the impedance eduction model, although higher order modes are propagating in the impedance tube facility.
\end{abstract}

\section{Nomenclature}

$$
\begin{array}{ll}
{[A], A_{p}} & =\text { Global stiffness matrix and complex mode coefficient of sound source } \\
c_{0}, M_{0}, \rho_{0} & =\text { speed of sound, uniform flow Mach number, and mean flow density } \\
f, k & =\text { frequency of sound source, freespace wavenumber } \\
\{P\},\{F\} & =\text { vector of unknown nodal coefficients and vector containing source effects } \\
H, L, W & =\text { height, length, and width of 3D duct } \\
i & =\sqrt{-1}, \text { unit imaginary number } \\
L_{1}, L_{2} & =\text { location of leading and trailing edge of test liner } \\
p g 0, p g L & =\text { the normal component of the acoustic pressure gradient at the source and exit plane nodes } \\
p, t & =\text { acoustic pressure field in duct and time } \\
X, Y & =\text { unconstrained optimization variables } \\
x, y, z & =\text { Cartesian coordinates } \\
\beta, \zeta & =\text { dimensionless acoustic admittance and impedance of test liner } \\
\theta, \chi & =\text { dimensionless acoustic resistance and reactance of test liner } \\
\phi(\zeta),\|\| & =\text { objective function, complex absolute value, and circular frequency } \\
\omega & =2 \pi f, \text { circular frequency }
\end{array}
$$

\footnotetext{
${ }^{*}$ Senior Research Scientist, Research and Technology Directorate, Computational AeroSciences Branch, Liner Physics Group, AIAA Associate Fellow.

${ }^{\dagger}$ Senior Research Scientist, Research and Technology Directorate, Structural Acoustics Branch, Head Liner Physics Group, AIAA Associate Fellow.
} 


$$
\begin{array}{ll}
\text { Subscripts: } & \\
I, J & =\text { counter for lower wall and source plane microphones } \\
\text { Meas }, s, t & =\text { a measured quantity, source plane quantity, and exit plane quantity } \\
\min , \max & =\text { minimum and maximum value of a quantity }
\end{array}
$$

\section{Introduction}

As part of NASA's Fundamental Aeronautics Program, focused efforts are currently underway to develop concepts to confine subsonic aircraft objectionable noise within the boundaries of the airport. Although acoustic liners mounted in the aircraft engine nacelles provide a significant portion of the current fan-noise reduction, they must be further optimized if this goal is to be achieved. This will require more detailed understanding of the locally-reacting acoustic impedance of nacelle liners in the presence of grazing flow. This impedance is an intrinsic property of the nacelle liner and is defined as the ratio of the acoustic pressure to the normal component of acoustic particle velocity at the surface of the liner. After several decades of research in impedance measurement technology, accurate and reliable tools for simultaneously measuring both the acoustic pressure and the normal component of acoustic particle velocity at the surface of a nacelle liner have proved challenging. Therefore a direct and reliable measurement of the locally-reacting impedance of a nacelle liner (in the presence of grazing flow) is not currently available.

As an alternative to direct measurement of the locally-reacting impedance, various numerical methods (which are used in conjunction with measurements from experimental facilities) have been developed. The numerical methods ${ }^{1-15}$ include codes based on single mode, multi-mode, and finite element analyses. Of these, finite element codes developed at the NASA Langley Research Center (LaRC) have been thoroughly tested in the open duct acoustics literature, $, 5,11,13$ and have been demonstrated to provide quality results in ducts where a single plane-wave mode can be isolated between one pair of opposite rigid walls. A number of experimental test rigs have also been developed to obtain measured data that can be used in conjunction with the numerical methods. Of particular note are the Spirit Aerosystems $^{6}$ (formerly a Boeing facility) and United Technology Research Center ${ }^{15}$ flow impedance tubes, and the Goodrich Aerostructures ${ }^{6}$ insertion loss facility. Further, over the last three decades, LaRC has developed a number of test rigs for the evaluation of locally-reacting acoustic liners in the presence of grazing flow. The most notable of these are the Grazing Incidence Tube ${ }^{16}$ (GIT) and the Curved Duct Test Rig (CDTR). ${ }^{17}$

In 2009 the GIT was updated with a rectangular cross-section and more advanced technology that improves the accuracy of the aeroacoustic and mean flow measurements. This new rig was brought online in 2009 and is referred to as the grazing flow impedance tube (GFIT). A schematic of the GFIT is given in Fig. 1. Impedances are educed for test liners in the GFIT using a procedure that has been well tested in the GIT. This procedure ${ }^{13}$ uses the acoustic pressure measured by microphones flush-mounted in the rigid wall opposite the test liner to educe the impedance and is referred to in this paper as the wall pressure method (WPM). It was clear from the onset of the current effort that the WPM might not be suited for application to a) test rigs that are much larger than the GFIT, b) test rigs that contain liners on opposite walls, and c) test rigs that contain wall curvature. The LaRC CDTR is an example of a test rig for which all of the above conditions are true. The reasons for these observations are as follows:

1. More microphones are required to implement WPM in larger ducts. Thus, there will be a need to reduce the number of microphones in larger ducts to control the cost of impedance eduction.

2. When two opposite walls in the liner test section are lined, then WPM would require that the fixed microphone array be installed into one of the rigid walls adjacent to the test liners. However, for curved ducts, the location of the fixed microphones will depend on the duct curvature and flow path. Thus, the fixed array will have to be removed and a new array of microphones inserted and recalibrated for each duct curvature under investigation. Thus, the WPM will add substantial cost and time to impedance eduction when used in curved duct configurations.

3. It is convenient to use the existing microphones in the hardwall sections of duct upstream and downstream of the test liner for impedance eduction.

In a recent paper, ${ }^{18}$ the authors presented a new numerical procedure for educing the impedance of test liners located in a duct with grazing flow. This procedure has the advantages that it is applicable to ducts containing nonplanar sources and with test liners on more than one wall as illustrated in Fig. 2. In addition, it does not require the use of a microphone array in the liner test section. Because this new method uses the acoustic pressure gradient normal to the source and exit boundary to educe the impedance, it is referred to in this paper as the pressure gradient method 
(PGM). The success of the PGM has been demonstrated using source and termination plane data that was obtained from an exact solution. ${ }^{18}$ However, there has been no validation of PGM using synthesized or measured data.

This paper has two primary objectives. The first objective is to describe and implement several enhancements to the $\mathrm{WPM}^{4}$ and $\mathrm{PGM}^{18}$ impedance eduction procedures. These enhancements are designed to improve both the accuracy of the educed impedance and the robustness of the impedance eduction procedures. The second objective is to make an initial attempt at validating the PGM using (1) data synthesized from a numerical method, and (2) measured data from the newly designed GFIT. The validation is achieved by comparing the educed impedance (or admittance, for a hardwall insert) for the WPM and the PGM over a range of frequencies and mean flow Mach numbers, using both a hardwall insert and a conventional test liner.

\section{Governing Equations and Boundary Conditions}

A schematic of the GFIT is given in Fig. 1. An orthogonal coordinate system is used in which $z$ is the axial coordinate and the two transverse coordinate are denoted by $x$ and $y$. The source and termination planes of the duct are located at $z=0$ and $z=L$, respectively. The upper wall contains the test liner and the remainder of the duct is rigid. The leading and trailing edge of the liner are located at $z=L_{1}$, and $z=L_{2}$, respectively, so that the liner is $\left(L_{2}-L_{1}\right)$ units in length. Note that the lower wall of the duct contains a number of flush-mounted microphones. Acoustic pressure data from these flush-mounted microphones are needed to educe the impedance using WPM. Data acquired at these microphones is therefore referred to as the WPM impedance eduction data. It is shown later how to use the flush-mounted lower wall microphones to approximate eduction data for the PGM. Under certain simplifying assumptions, the acoustic pressure field in the GFIT (see the duct in Fig. 1) can be shown to satisfy the 2D convected Helmholtz equation

$$
\left(1-M_{0}^{2}\right) \frac{\partial^{2} p(z, x)}{\partial z^{2}}+\frac{\partial^{2} p(z, x)}{\partial x^{2}}-2 i k M_{0} \frac{\partial p(z, x)}{\partial z}+k^{2} p(z, x)=0
$$

where a time-harmonic dependence of the form $e^{i \omega t}$ has been assumed. The simplifying assumptions made in deriving the convected Helmholtz equation are

1. the flow in the duct can be decomposed into an acoustic and mean flow field, and the mean velocity field has only a uniform axial component

2. the acoustic field is linear, time-periodic, not affected by viscous forces, and small compared to the mean flow

3. the acoustic pressure and density are adiabatically related

4. the source frequency, $f$, is sufficiently low that only plane acoustic waves are in the $y$ transverse direction

All of the simplifying assumptions above are reasonable inside of a duct with large cross-sectional dimensions such as CDTR. However, the assumption of a uniform flow field may be difficult to achieve at the higher flow speeds inside of a duct with small cross-sectional dimensions such as the GFIT. For a detail derivation of the convected Helmholtz equation, readers are referred to Ref. 19.

The convected Helmholtz equation is solved subject to the source plane, termination plane, and lower wall boundary conditions

$$
p(0, x)=p_{s}, \quad p(L, x)=p_{t}, \quad \frac{\partial p(z, 0)}{\partial x}=0
$$

The locally reacting, wall impedance boundary condition at the surface of the liner has been derived by Myers. ${ }^{20}$ This expression relates the normal component of acoustic particle velocity to the acoustic pressure field at the surface of the liner. When written in terms of the acoustic pressure field, the locally reacting wall impedance boundary condition is

$$
-\frac{\partial p(z, H)}{\partial x}=i k\left[\frac{p(z, H)}{\zeta}\right]+2 M_{0} \frac{\partial}{\partial z}\left[\frac{p(z, H)}{\zeta}\right]+\frac{M_{0}^{2}}{i k} \frac{\partial^{2}}{\partial z^{2}}\left[\frac{p(z, H)}{\zeta}\right]
$$

In Eq. (3), $\zeta=\theta+i \chi$, is the unknown dimensionless impedance of the test sample and its admittance, $\beta$ (i.e., $\beta=1 / \zeta$ ), is taken as zero along the rigid portion of the upper wall. The parameters $\theta$ and $\chi$ are the unknown dimensionless resistance and reactance, respectively, of the test liner. Throughout this paper all resistances and reactances are made dimensionless by dividing by the characteristic impedance, $\rho_{0} c_{0}$, of an outgoing plane wave in an equivalent hardwall duct. 
For a specified wall impedance, $\zeta$, Eqs. (1)-(3) constitute a boundary value problem (BVP) that can be solved numerically to determine a unique set of lower wall acoustic pressures, $p\left(z_{I}, 0\right)$, at the flush-mounted microphone locations (i.e., $\left.z=z_{I}\right){ }^{19}$ Similarly, the solution to the same boundary value problem can be solved to determine unique values of the acoustic pressure gradients $\left(\frac{\partial p\left(0, x_{J}\right)}{\partial z}\right.$ and $\left.\frac{\partial p\left(L, x_{J}\right)}{\partial z}\right)$ normal to the source and exit boundary. For a uniqueness proof of the solution to the BVP, readers are referred to Ref. 19. In the above discussion, the notation, $x_{J}$, is used to denote a transverse position on the source and exit boundary where the pressure gradients normal to a boundary are measured. It is noted that the uniqueness of the lower wall acoustic pressure profile from the solution of the $\mathrm{BVP}^{19}$ provides the basis for the WPM impedance eduction method. ${ }^{18}$ Similarly, the uniqueness of the normal pressure gradients at the source and exit planes from the solution to the BVP provides the basis for the PGM impedance eduction method. ${ }^{13}$

\section{Brief Description of the Impedance Eduction Techniques}

The technique used to educe the impedance parallels that used in two earlier papers. ${ }^{4,18}$ Therefore only a brief description of the impedance eduction techniques is provided in the current paper. Both impedance eduction techniques are similar and may be summarized as follows:

1. Measure the mean flow data (i.e., $\left.M_{0}, \rho_{0}, c_{0}\right)$

2. Measure the source and termination plane acoustic pressures $p_{\mathrm{s}}$ and $p_{\mathrm{t}}$. Both $p_{s}$ and $p_{t}$ are assumed planar for the current application. This assumption is made because the GFIT was not designed to measure the acoustic energy in higher order duct modes. However, there is no requirement in the preceding or the following discussions that either $p_{s}$ or $p_{t}$ be planar (i.e., independent of the coordinate $x$ ).

3. Measure the impedance eduction data which is different for WPM and PGM

(a) For the WPM, measure the acoustic wall pressures along the array of flush-mounted wall microphones

(b) For the PGM, measure the component of the acoustic field necessary to determine the component of the acoustic pressure gradient normal at the source and exit planes.

4. Using an initial guess for the test liner impedance, $\zeta$, solve the BVP defined by Eqs. (1)-(3) to obtain a unique set of lower wall acoustic pressures (for the WPM impedance eduction methodology) or a unique set of acoustic pressure gradients normal to the source and exit boundaries (for the PGM impedance eduction methodology).

5. Compare the measured data (i.e, the measured lower wall acoustic pressure for the WPM and the measured values of the source and exit plane acoustic pressure gradients for the PGM) to that computed from the solution to the BVP. If the computed and measured values agree (to within some predetermined tolerance), then the initial guess for the test liner impedance is the impedance of the test liner.

6. If the computed and measured values do not agree, then the unknown impedance, $\zeta$, of the test liner is iterated upon until the measured and numerically computed values match (to within the predetermined tolerance). The resulting impedance is the unknown impedance of the test liner.

It is convenient to formulate both of the above impedance eduction techniques as optimization problems. That is, a value of the impedance is sought that minimize the objective function, $\phi$. This objective function has a different form depending on whether the WPM or the PGM eduction procedure is used. For the WPM, the objective function is defined as the L2 norm of the difference between the measured lower wall acoustic pressure and the numerical solution obtained from the solution to the BVP

$$
\phi(\zeta)=\sqrt{\sum_{I=1}^{I=N}\left\|p_{\text {Meas }}\left(z_{I}\right)-p\left(z_{I}, 0\right)\right\|}
$$

For the PGM, the objective function is defined as the L2 norm of the difference between the measured and the numerical solution for the acoustic pressure gradient normal to the source and the exit plane

$$
\phi(\zeta)=\sqrt{\sum_{I=1}^{I=M}\left\|p g 0_{\text {Meas }}\left(x_{J}\right)-\frac{\partial p\left(0, x_{J}\right)}{\partial z}\right\|+\left\|p g L_{\text {Meas }}\left(x_{J}\right)-\frac{\partial p\left(L, x_{J}\right)}{\partial z}\right\|}
$$


Here, $p g 0_{\text {Meas }}\left(x_{J}\right)$ and $p g L_{\text {Meas }}\left(x_{J}\right)$ are the measured normal component of the acoustic pressure gradient along the source and exit planes, respectively. Note that $p g 0_{\text {Meas }}\left(x_{J}\right)$ and $p g L_{\text {Meas }}\left(x_{J}\right)$ are the eduction data for PGM.

In the current implementation of the optimization method, the numerical solution to the BVP is obtained using a cubic finite element methodology (FEM). ${ }^{13}$ The FEM leads to a large but sparse matrix equation of the form

$$
[A]\{P\}=\{F\}
$$

where the solution vector $\{P\}$ provides the unique set of lower wall acoustic pressures (i.e., $p\left(z_{I}, 0\right)$ ) for the WPM impedance eduction methodology, or the unique set of pressure gradients normal to the source and exit planes (i.e., $\frac{\partial p\left(0, x_{J}\right)}{\partial z}$ and $\left.\frac{\partial p\left(L, x_{J}\right)}{\partial z}\right)$ for the PGM. Readers are reminded that the BVP that is solved for the WPM and PGM are identical. Only the data used to post-process the solution and construct the objective function is different for the two impedance eduction methodologies. The optimization algorithm chosen to minimize the objective function is Stewart's adaptation of the Davidon-Fletcher-Powell (SDFP) optimization algorithm. ${ }^{21}$ This is a gradient-based optimizer that uses a finite difference approximation to obtain the gradient of the objective function and is chosen because of its computational efficiency.

\section{Enhancements to the Impedance Eduction Methods}

Several enhancements to the original impedance eduction methods ${ }^{4,18}$ have been implemented in this paper. These are described in the following subsections.

\section{A. Accuracy Improvements}

One lesson learned in a previous study ${ }^{13}$ is that the accuracy of the computational model is increased by switching from an exit impedance boundary condition at $z=L$, to the exit pressure boundary condition defined by Eq. (2). This increase in accuracy of the computational model occurs because a direct measurement of the exit pressure, $p_{s}$, is available to implement into the exit pressure boundary condition (see Eq. (2)). However, the exit impedance needed for the exit impedance boundary condition is not directly measurable. Therefore the exit impedance is approximated from the measured lower wall pressures. The accuracy of the solution vector, $\{P\}$, from Eq. (6) is also improved by switching from the SGI to the INTEL sparse equation solver and then performing iterative refinement. The SGI solver that was used in earlier papers does not provide the capability to improve the solution vector by iterative refinement.

\section{B. Use of a Constrained Optimization}

The SDFP algorithm is defined only for unconstrained optimization problems. Subsequently, the educed resistance can sometimes converge to a negative value. Physically, a negative resistance implies that there is another active source of sound in the duct other than $p_{s}$ or that the background noise level in the duct is above that of the sound source. The capability to constrain both the dimensionless resistance and reactance has been implemented into the current study. The constraints are implemented as described below. First, let the unconstrained design variables used by SDFP be denoted by $X$ and $Y$, and let the resistance and reactance to be constrained be defined as:

$$
\begin{aligned}
& \theta=\theta_{\text {min }}+\left(\theta_{\text {max }}-\theta_{\text {min }}\right) \sin ^{2}(X) \\
& \chi=\chi_{\text {min }}+\left(\chi_{\text {max }}-\chi_{\text {min }}\right) \sin ^{2}(Y)
\end{aligned}
$$

In Eq. (7), $\theta_{\min }$ and $\theta_{\max }$ are the minimum and maximum values, respectively, of the dimensionless resistance, $\theta$, in the constrained design space. Likewise, in Eq. (8), $\chi_{\min }$ and $\chi_{\max }$ are the minimum and maximum values, respectively, of the dimensionless reactance, $\chi$, in the constrained design space. The unconstrained optimization algorithm (SDFP) is now run with $X$ and $Y$ as the unconstrained design variables. To constrain the dimensionless resistance and reactance to ranges expected for the majority of liners to be tested in the GFIT, the minimum and maximum values are chosen as follows:

$$
\begin{gathered}
\theta_{\max }=\chi_{\max }=10 \\
\theta_{\min }=0, \quad \chi_{\min }=-10
\end{gathered}
$$




\section{Use of Multiple Starting Locations}

Because local optimizers such as SDFP can converge to local optima, the following strategy is used to increase the likelihood that a global optimum is achieved:

1. The constrained designed space is divided into four quadrants (quadrant I, quadrant II, quadrant III, and quadrant IV) that are determined as follows:

(a) For quadrant I : $\theta_{\min } \leq \theta \leq \frac{1}{2}\left(\theta_{\max }+\theta_{\min }\right)$, and $\chi_{\min } \leq \chi \leq \frac{1}{2}\left(\chi_{\max }+\chi_{\min }\right)$

(b) For quadrant II: $\frac{1}{2}\left(\theta_{\max }+\theta_{\min }\right) \leq \theta \leq \theta_{\max }$, and $\chi_{\min } \leq \chi \leq \frac{1}{2}\left(\chi_{\max }+\chi_{\min }\right)$

(c) For quadrant III: $\frac{1}{2}\left(\theta_{\max }+\theta_{\min }\right) \leq \theta \leq \theta_{\max }$, and $\frac{1}{2}\left(\chi_{\max }+\chi_{\min }\right) \leq \chi \leq \chi_{\max }$

(d) For quadrant IV: $\theta_{\min } \leq \theta \leq \frac{1}{2}\left(\theta_{\max }+\theta_{\min }\right)$, and $\frac{1}{2}\left(\chi_{\max }+\chi_{\min }\right) \leq \chi \leq \chi_{\max }$

2. SDFP is run four times using the midpoint of each quadrant as the starting location

3. If the impedances obtained from each of the four starting locations are identical, then this impedance is taken as the impedance of the test liner

4. If the impedances obtained from each of the four runs are not identical, then the impedance with the smallest objective function is taken as the impedance of the test liner. The rationale for this decision is that the impedance with the smallest objective function is the one that best matches the experimental data (see Eqs. (4) and (5)).

\section{Results and Discussion}

An in-house computer code that implements the enhancements to the WPM and PGM impedance eduction techniques has been developed. The code uses F90 Fortran with double precision (i.e., 64 bit) arithmetic and was designed to run in a shared memory environment. This approach has been chosen because the primary hardware to be utilized is a shared memory system. In the current implementation, the resistance, $\theta$, and reactance, $\chi$, of the test liner are the design variables for the constrained optimization and the four starting locations discussed in the previous subsection are used.

The computational cost of the impedance eduction grows as the FEM spatial grid increases in density. Prior to impedance eductions, a grid refinement study was conducted at each frequency of interest to determine the coarsest grid for accurate impedance eductions (these grid refinement studies are not presented for the sake of brevity). Four separate finite element grids (each obtained from doubling of a coarser grid) were investigated. The FEM spatial grid used in the results to follow was an $161 \times 26$ evenly spaced spatial grid, where the largest number of points (i.e., 161) was along the axis of the duct. This was the second coarsest grid used in the grid refinement study for which the educed impedance was observed to have converged.

Several examples are presented in this section to validate the PGM by comparing it to the well tested WPM . Results are presented for three sets of eduction data: (1) eduction data acquired from an analytical solution, (2) eduction data acquired from a FEM solution to the BVP, and (3) eduction data acquired from measurements in the GFIT. The duct geometry is that of the GFIT for which $L_{1}=203 \mathrm{~mm}, L_{2}=601 \mathrm{~mm}, L=1061 \mathrm{~mm}, W=5.08 \mathrm{~mm}$, and $H=6.35 \mathrm{~mm}$ (see Fig. 1). Source plane, termination plane, and mean flow data are simulated (or measured) at three centerline Mach numbers $(0.0,0.3$, and 0.5$)$, for an incident sound pressure level of $130-\mathrm{dB}$, and for source frequencies ranging from 0.4 to $3.0 \mathrm{kHz}$ in increments of $0.2 \mathrm{kHz}$. For the WPM, 53 unevenly spaced flush-mounted wall microphones are used to acquire eduction data. This is the arrangement currently used in the GFIT. The 53 microphones are allocated in the lower rigid wall of the GFIT so that :

1. nine microphones are located upstream of the leading edge of the liner

2. 35 microphones are located in the portion of the wall opposite the test liner

3. the remaining nine microphones are located in the rigid wall section downstream of the trailing edge of the liner.

For the PGM, 26 evenly spaced transverse locations are used to acquire eduction data. Finally, results are presented for two test liners. The first test liner is a hardwall insert and the second is a conventional test liner consisting of a perforated plate bonded to a honeycomb core. 


\section{A. Hardwall Insert}

The hardwall insert is a $12.7 \mathrm{~mm}$-thick stainless steel plate. The hardwall insert provides a liner for which the dimensionless admittance, $\beta=1 / \zeta$, is known. The eduction data required by WPM and PGM for the hardwall insert was acquired in two ways. First, the admittance of the liner is set to that of a perfect hardwall (i.e., $\beta=0$ ) and the eduction data is acquired from the analytical solution for an outgoing plane wave in an infinitely long hardwall duct

$$
p(z, x)=A_{p} e^{-i k z}
$$

Here, the complex mode coefficient, $A_{p}$, is chosen so that the sound pressure level at the source plane is $130 \mathrm{~dB}$. Next, the hardwall insert is inserted into the GFIT and the WPM eduction data is measured using the 53 flush-mounted wall microphones. Because the GFIT was not designed to perform a direct measurement of the eduction data for the PGM, this data was acquired using a one-sided difference. That is, the acoustic pressure gradient normal to the source plane was approximated using the forward difference expression

$$
\frac{\partial p}{\partial z}=\left[p_{\text {Meas }}\left(z_{2}\right)-p_{\text {Meas }}\left(z_{1}\right)\right] /\left(z_{2}-z_{1}\right)
$$

while at the exit plane, the expression used is the backward difference expression

$$
\frac{\partial p}{\partial z}=\left[p_{\text {Meas }}\left(z_{N}\right)-p_{\text {Meas }}\left(z_{N-1}\right)\right] /\left(z_{N}-z_{N-1}\right)
$$

where the source and exit planes are located at $z=z_{1}$ and $z=z_{N}$, respectively. The microphones located at $z=z_{1}$ and $z=z_{2}$ are the two furthest upstream microphones and are $2.54 \mathrm{~cm}$ apart. Similarly, the microphones located at $z=z_{N}$ and $z=z_{N-1}$ are the two furthest downstream microphones, and they are also $2.54 \mathrm{~cm}$ apart.

The correct dimensionless conductance $(\operatorname{Real}[\beta])$ and susceptance $($ Imaginary $[\beta])$ for a rigid test sample are known to be zero. Figure 3 compares the educed dimensionless conductance and dimensionless susceptance for the WPM and PGM at Mach 0.0, when the analytical eduction data (see Eq. (11)) is used. The dimensionless conductance and susceptance are shown on a dual axis system with the dimensionless conductance referenced to the left axis and the dimensionless susceptance referenced to the right axis. Each impedance eduction method produces the correct value of zero for the dimensionless conductance and susceptance, except at 1.4 and $1.6 \mathrm{kHz}$. A contour plot of the objective function (not shown in this report for the sake of brevity) shows that the gradient of the objective function is too steep for SDFP at these two frequencies and this is the source of the discrepancy. Recall that SDFP uses a finite-difference expression to approximate the gradient of $\phi(\zeta)$ and that approximation will not be accurate for an extremely steep gradient. Note that the PGM is as robust and accurate as the WPM (see Fig. 3). Results for Mach 0.3 using the analytical eduction data are shown in Fig. 4. The educed dimensionless conductance and susceptance are in excellent agreement with their expected value of zero, except at $0.6 \mathrm{kHz}$, where the gradient of the objective function was again found to be too steep for SDFP to resolve. The educed dimensionless conductance and susceptance spectrum at the highest Mach number (i.e., Mach 0.5) are given in Fig. 5. Educed dimensionless conductance and susceptance values at this highest Mach number are also observed to match the expected value of zero. However, there is a discrepancy in the educed admittance at a few more frequencies (i.e., 0.8, 1.4, and $1.6 \mathrm{kHz}$ ) than for the lower Mach numbers. The steepness of the objective function at these frequencies caused SDFP to educe incorrect values for the dimensionless susceptance for both WPG and PGM at this highest Mach number. This was observed by looking at a contour plot of the objective function. It is interesting that only the educed susceptance seems to be negatively affected by the steep gradients in the objective function.

Figures 6-8 give plots of the educed dimensionless conductance and susceptance spectra (using the measured data) for the hardwall insert at Mach numbers of 0.0, 0.3, and 0.5, respectively. Several consistent trends are observed:

1. Both methods (WPM and PGM) educed the correct dimensionless conductance across the Mach number range except for an extremely small combination of Mach number and frequencies.

2. The PGM is slightly better at educing the correct dimensionless conductance across the frequency spectrum than the WPM. This results is rather surprising, in view of the fact that a one-sided difference is used to approximate the PGM eduction data from the measured acoustic wall pressures.

3. Both eduction methods do well at educing the expected dimensionless susceptance except in the mid-frequency range (where both encounter extremely sharp gradients in the objective function and SDFP gives incorrect results). Outside of this mid-frequency range, both the WPM and PGM educe nearly the same dimensionless susceptance spectrum. Additional evidence (not presented in this paper) suggest that the some of the sharp peaks in this mid-frequency range may be reduced by the inclusion of the mean boundary layer. 


\section{B. Conventional Test Liner}

The impedance of the conventional test liner is first obtained from an impedance prediction model. The inputs to the impedance prediction model are the liner geometry (i.e., hole size, facesheet thickness, cavity depth, porosity, etc.) and mean flow Mach number. Because the equations are programmed into a digital computer, the model is referred to here as the computer prediction model (CPM). Once the impedance is obtained by CPM, the eduction data for WPM and PGM is synthesized by running the finite element duct propagation code using the impedance obtained from the CPM. This validation exercise is performed to determine if the educed impedance for the conventional liner from WPM and PGM match that from the CPM. Near perfect agreement is expected between CPM, WPM, and PGM because the eduction data is synthesized from the solution to the FEM model.

The educed dimensionless resistance and reactance of the conventional test liner (using synthesized eduction data from the FEM model) for a mean flow Mach number of zero is given in Fig 9. Note that the impedance for this liner has a low resistance across the frequency range and the reactance shows a $-\cot (k d)$ behavior (as expected). Here, $d$ is the depth of the cavity. The WPM and PGM are observed to educe the identical dimensionless resistance and reactance spectrum as the CPM. Results for Mach 0.3 and 0.5 are given in Figs. 10 and 11, respectively. Both the WPM and PGM are observed to be equally accurate when used with the synthesized eduction data. Both methods are also observed to be equally robust.

In our final validation exercise, the conventional liner is inserted into the GFIT and the eduction data for the WPM and PGM are based on measured data. The PGM eduction data is acquired by applying one-sided differences to the measured lower wall acoustic pressure just as in the hardwall example. Readers are reminded that in the softwall example, there will be scattering of acoustic energy into higher-order modes at the leading and trailing edges of the liner. This scattering may be further enhanced by the refracted effects of the mean boundary layer which is present in the measurement, although it was neglected in the FEM model. Figure 12 compares the educed resistance and reactance spectra obtained using the PGM and WPM for the conventional liner without flow (i.e., $M_{0}=0.0$ ). These zero-flow comparisons are extremely encouraging. Note that the educed WPM and PGM dimensionless resistance and reactance are in very good agreement with each other except in the middle of the frequency range (i.e., 1.6 and $1.8 \mathrm{kHz}$ ) and for frequencies beyond $2.7 \mathrm{kHz}$. At 1.6 and $1.8 \mathrm{kHz}$ the objective function was extremely flat and the results returned by SDFP were highly sensitive to the initial starting location. Differences in the educed impedance at frequencies beyond $2.7 \mathrm{kHz}$ were expected. It should be noted that $2.7 \mathrm{kHz}$ is the cut on frequency of higher-order duct modes in the GFIT, and these higher-order modes have been neglected in the source and exit plane boundary conditions of the FEM model. Note also that at $3.0 \mathrm{kHz}$, the SDFP algorithm is stuck on the constrained boundary of the domain for the PGM. This often happens with constrained optimizations and although remedies exist for correcting this situation, these remedies may be futile without including the effects of the higher-order cut on modes that propagate at this frequency. Note that when one excludes the outliers (that have been explained previously) the educed impedance has the expected trends. That is, the educed impedance is a low resistance liner with a dimensionless resistance spectrum that is nearly independent of frequency and the dimensionless reactance follows a $-\cot (k d)$ behavior. The educed resistance and reactance spectrum for Mach 0.3 and Mach 0.5 (using the measured data) are given in Figs. 13 and 14, respectively. Good comparison between the PGM and WPM is again observed except for a small number of frequencies in the mid-frequency range where the objective function is extremely flat, and for frequencies beyond which higher-order modes are cut on in the GFIT but not accounted for in the FEM.

\section{Closing Remarks and Conclusions}

Several enhancements to an impedance eduction technique that improves the accuracy of the educed impedance and the robustness of the impedance eduction procedures have been presented. These enhancements have been used in an initial attempt to validate the pressure gradient method (PGM) of impedance eduction. This has been achieved by comparing the educed impedance spectrum to that of the more commonly used wall pressure method (WPM) for a hardwall and a conventional test liner. Validation exercises were carried out using (1) analytical eduction data, (2) eduction data synthesized from a finite element (FEM) solution, and (3) eduction data acquired frorm the LaRC Grazing Flow Impedance Tube (GFIT). The following primary conclusions are drawn from the results of this study:

1. For the hardwall insert

(a) When used with analytical eduction data, the educed admittance spectra, for the WPM and PGM are in very good agreement with the known admittance spectrum except for a relatively small combination of Mach number and frequencies. At these combinations of Mach numbers and frequencies, the objective 
function is extremely sharp. Although the number of these sharp peaks increases with increasing Mach number, only the educed susceptance is negatively impacted by these sharp peaks. When compared to the admittance spectrum educed by the WPM, the PGM is just as accurate and robust.

(b) When used with measured eduction data, the educed impedance spectrum for the PGM shows a slight improvement over that educed by WPM. This improvement shows up primarily in the educed conductance spectrum.

(c) When used with measured eduction data, both the WPM and PGM show some discrepancy in educed admittance in the mid-frequency range. This discrepancy occurs because the gradient of the objective function is too steep for the gradient-based optimizer. Indications are that this sharp gradient may be triggered by an inconsistency between the measured eduction data and the FEM model (the measured data contains the mean boundary layer effects and the FEM model does not).

2. For the conventional liner

(a) When used with data synthesized from the finite model, both the WPM and PGM educed the same impedance spectrum and both methods are equally accurate and robust.

(b) When used with measured data, the WPM and PGM educed impedance are in very good agreement with each other, except for one or two frequencies in the middle of the frequency range. Within this midfrequency range, the objective function is extremely flat and the educed impedance from SDFP is highly sensitive to the initial starting location. There is some spread in the WPM and PGM educed impedance for frequencies beyond the cut on frequency of higher-order duct modes. This spread appears to be due to a breakdown in the FEM model because the source and exit plane boundary condition is assumed to contain only plane waves. The resistance spectrum educed using both methods (WPM and PGM) is observed to be a low resistance liner that is nearly independent of frequency at Mach zero, and the reactance has a $-\cot (k d)$ behavior. The educed resistance spectrum is observed to increase with increasing Mach number and the reactance is observed to have a $-\cot (k d)$ behavior (as expected).

The success of the validation exercises conducted in this paper warrants additional studies of the PGM method using the CDTR. The CDTR offers the possibility of an improved measurement of the PGM eduction data, better control and measurement of higher-order duct mode content, and a mean flow that is much closer to uniform than that in the GFIT. Efforts are also underway to include the effects of the mean boundary layer into the PGM eduction method. This is expected to improve the prediction across the frequency spectrum.

\section{Acknowledgement}

This work was supported by the Subsonic Fixed Wing Project of NASA's Fundamental Aeronautics Program.

\section{References}

\footnotetext{
${ }^{1}$ Armstrong, D. L., Beckemeyer, R. J., and Olsen, R. F., “Impedance Measurements of Acoustic Duct Liners With Grazing Flow," Paper presented at the 87th Meeting of the Acoustical Society of America, New York, NY, 1974.

${ }^{2}$ Watson W. R., "A New Method for Determining Acoustic-Liner Admittance in Ducts with Sheared Flow in Two Cross-Sectional Directions," NASA TP-2518, 1985.

${ }^{3}$ Watson, W. R., Tanner, S. E., and Parrott, T. L., "Optimization Method for Educing Variable-Impedance Liner Properties," AIAA Journal, Vol. 36, No. 1, 1998, pp. 18-23.

${ }^{4}$ Watson, W. R., Jones, M. G., and Parrott, T. L., "Validation of an Impedance Eduction Method in Flow," AIAA Journal, Vol. 37, No. 7, pp. 818-824, July 1999.

${ }^{5}$ Watson, W. R., Tracy, M. B., Jones, M. G., and Parrott, T. L., "Impedance Eduction in the Presence of Shear Flow," AIAA Paper 2001-2236, May 2001.

${ }^{6}$ Jones, M. G., Parrott, T. L., and Watson, W. R., "Comparison of Acoustic Impedance Eduction Techniques for Locally-Reacting Liners," AIAA Paper 2003-3306, May 2003.

${ }^{7}$ Elnady, T., and Boden, H., "An Inverse Analytical Method for Extracting Liner Impedance from Pressure Measurements," AIAA Paper 2004-2836, May 2004.

${ }^{8}$ Jones, M. G., Watson, W. R., Tracy, M. B., and Parrott, T. L., "Comparison of Two Waveguide Methods for Educing Liner Impedance in Grazing Flow," AIAA Journal, Vol. 42, No. 2, 2004, pp. 232-240.

${ }^{9}$ Auregan, Y., Leroux, M., and Pagneux, V., “Measurement of Liner Impedance with Flow by an Inverse Method,” AIAA Paper 2004-2838, May 2004.
} 
${ }^{10}$ Watson, W. R., Jones, M. G., and Parrott, T. L., "A Quasi-3-D Theory for Impedance Eduction in Uniform Grazing Flow," AIAA Paper 2005-2848, May 2005.

${ }^{11}$ Watson, W. R., Jones, M. G., and Parrott, T. L., "Comparison of a Convected Helmholtz and Euler Model for Impedance Eduction in Flow," AIAA paper 2006-2643, May 2006.

${ }^{12}$ Elnady, T., Musharraf, M., Boden, H., and Elhadidi, B., "Validation of an Inverse Analytical Technique to Educe Liner Impedance with Grazing Flow," AIAA paper 2006-2639, May 2006.

${ }^{13}$ Watson, W., Jones, M., and Parrott, T., "Investigation of an Anomaly Observed in Impedance Eduction Techniques," AIAA paper 2008-3013, May 2008.

${ }^{14}$ Jing, X., Peng, S., and Sun, X., “A Straightforward Method for Wall Impedance Eduction in a Flow Duct,” J. Acoust. Soc. Am., Vol. 124, No. 1, July 2008.

${ }^{15}$ Simonich, J., Narayanan, S., Morin, B., and Patrick, W., "Development and Quantification of an In-Situ Grazing Flow Impedance Measurement Technique," AIAA Paper 2005-2848, May 2005.

${ }^{16}$ Jones, M. G., Watson, W. R., Parrott, T. L., and Smith, C. D., "Design and Evaluation of Modifications to the NASA Langley Flow Impedance Tube," AIAA Paper 2004-2837, May 2004.

${ }^{17}$ Gerhold, C., Cabell, R., and Brown, M., "Development of an Experimental Rig for Investigation of Higher Order Modes in Ducts," AIAA Paper 2006-2637, May 2006.

${ }^{18}$ Watson, W. R., and Jones, M. G, “Impedance Eduction in Ducts with Higher Order Modes and Flow," AIAA Paper 2009-3236, May 2009.

${ }^{19}$ Kraft, R. E., "Theory and Measurement of Acoustic Wave Propagation in Multi-Sectioned Rectangular Ducts," Ph.D. dissertation , Department of Aerospace Engineering, University of Cincinnati, Cincinnati Ohio, 1976, pp. 78-85.

${ }^{20}$ Myers, M. K., "On the Acoustic Boundary Condition in the Presence of Flow," Journal of Sound and Vibration, Vol. 71, No. 3, 1980, pp.429-434.

${ }^{21}$ Stewart, G. W. III, “A Modification of Davidon's Minimization Method to Accept Difference Approximations of Derivatives," Journal of ACM, Vol. 14, No. 1, 1967, pp. 72-83.

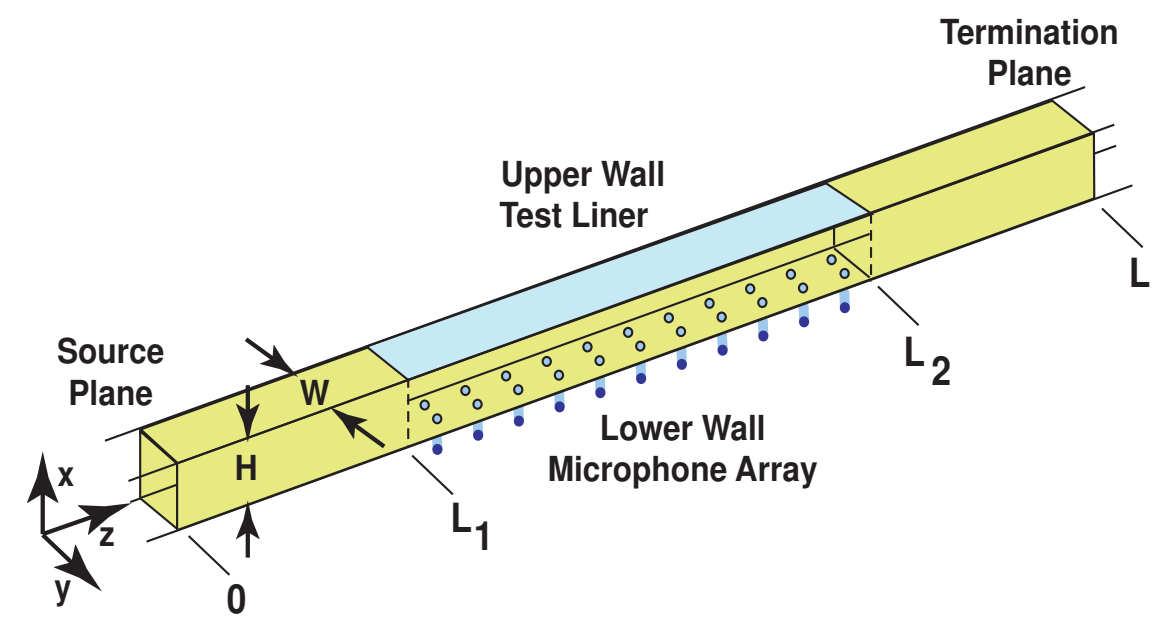

Figure 1. Schematic of the LaRC grazing flow impedance tube with microphones embedded in the liner test section. 


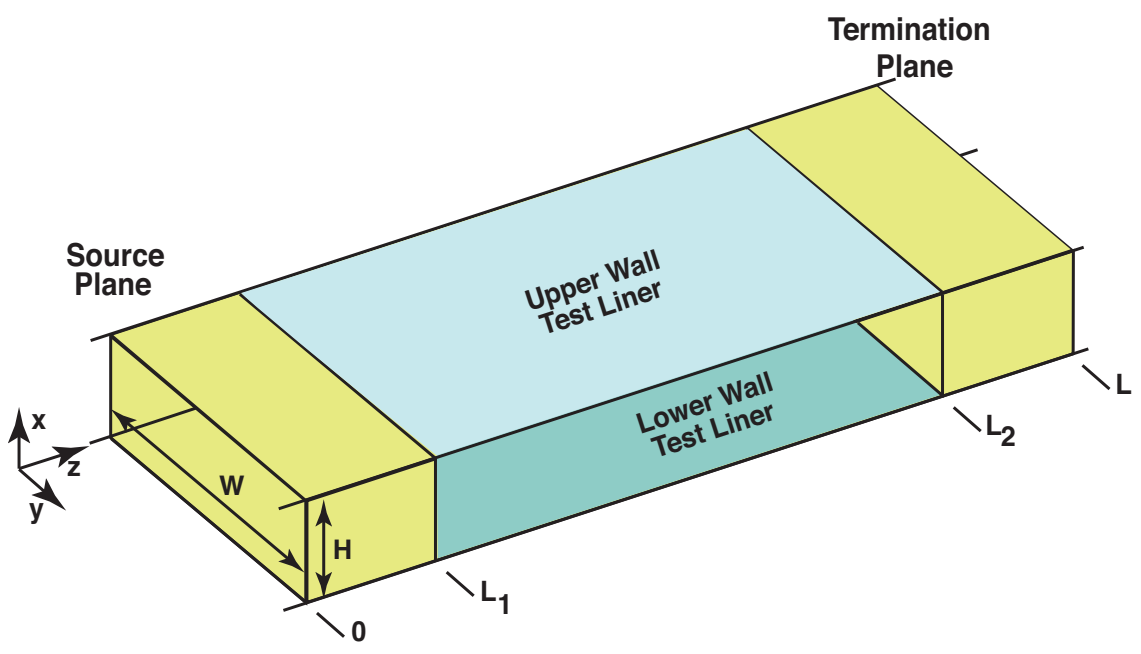

Figure 2. Schematic of 3D duct with microphones removed from liner test section.

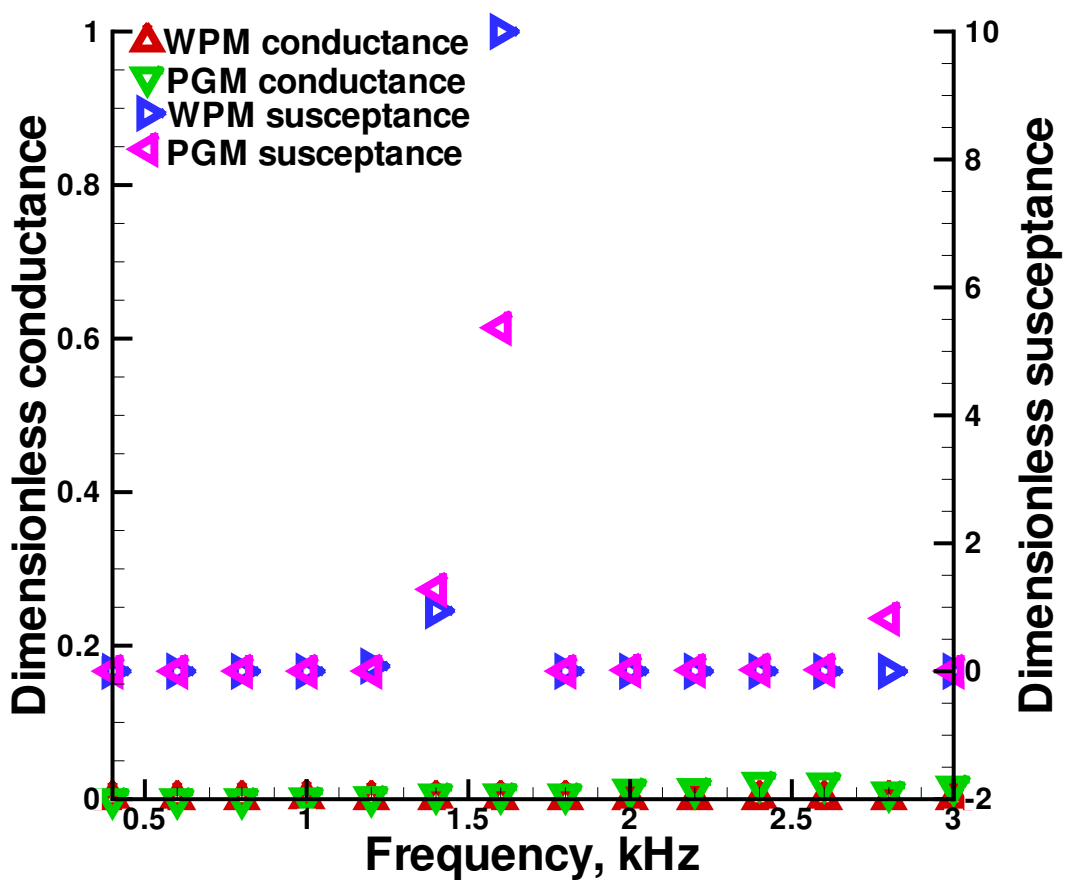

Figure 3. Educed dimensionless admittance of hardwall insert at Mach 0.0 (analytical eduction data). 


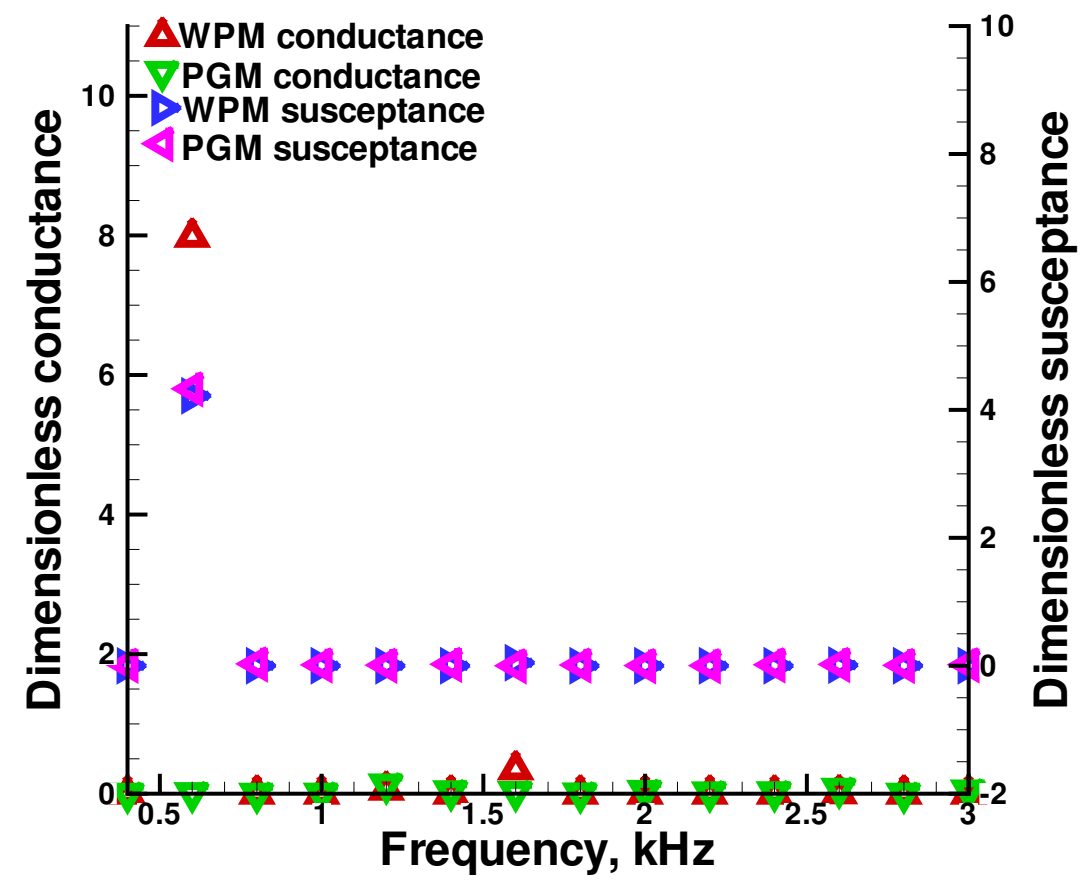

Figure 4. Educed dimensionless admittance of hardwall insert at Mach 0.3 (analytical eduction data).

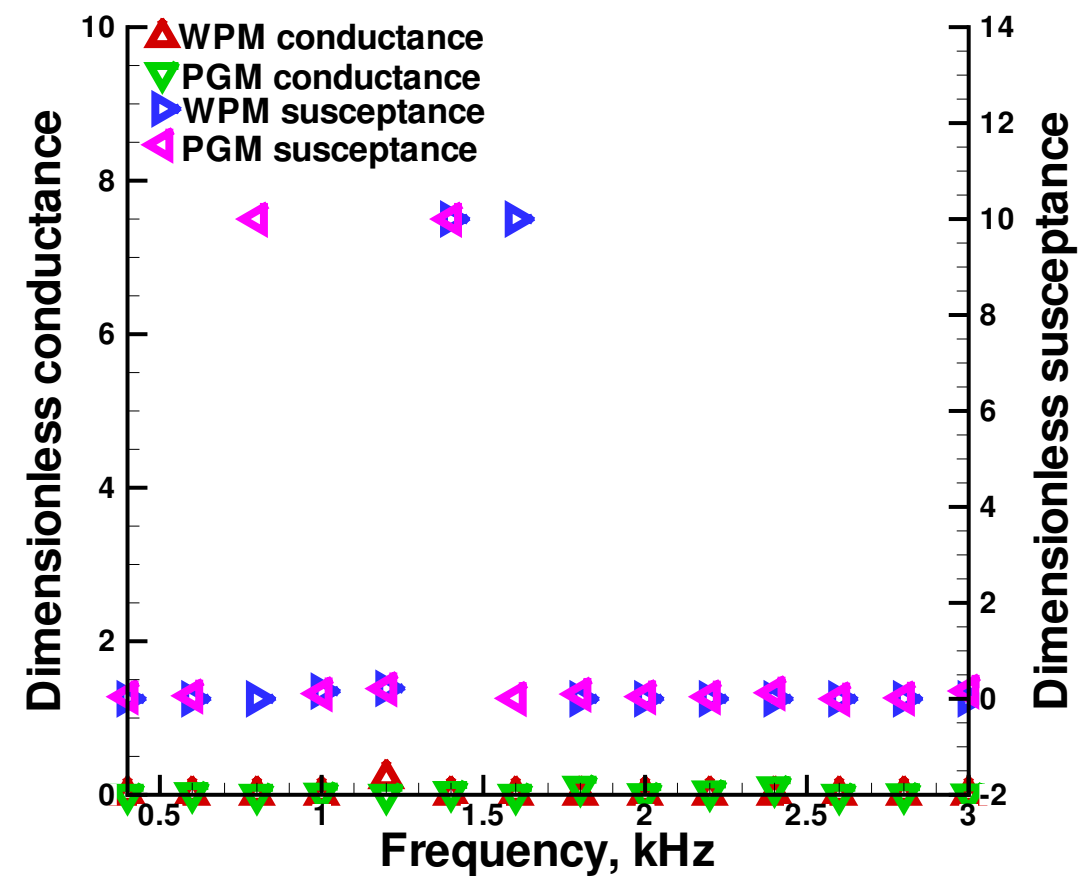

Figure 5. Educed dimensionless admittance of hardwall insert at Mach 0.5 (analytical eduction data). 


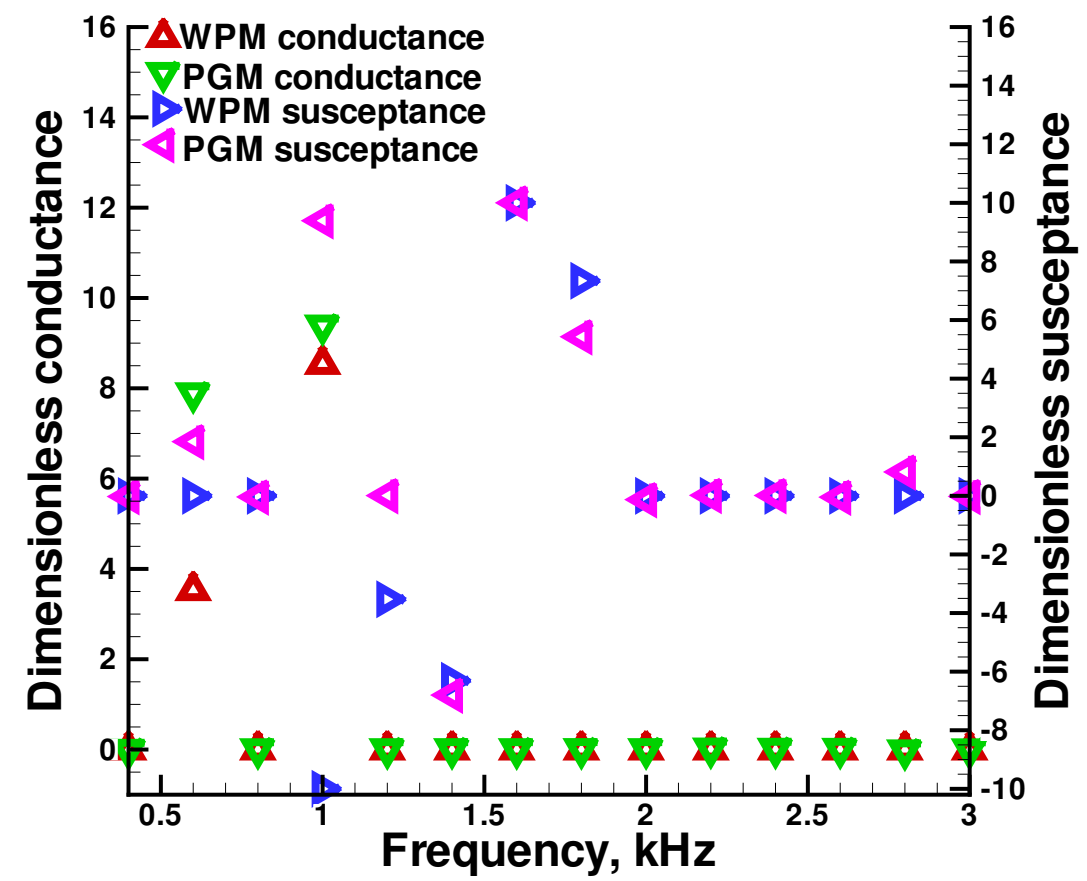

Figure 6. Educed dimensionless admittance of hardwall insert at Mach 0.0 (measured eduction data).

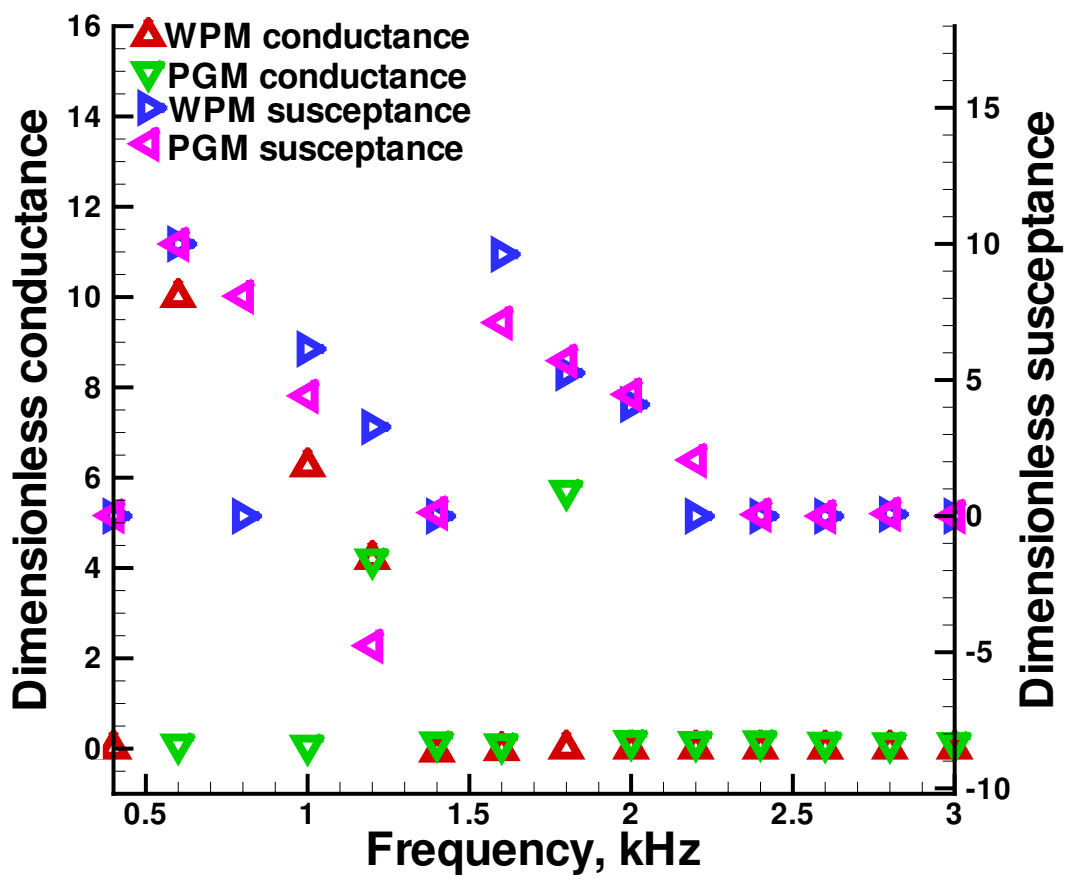

Figure 7. Educed dimensionless admittance of hardwall insert at Mach 0.3 (measured eduction data). 


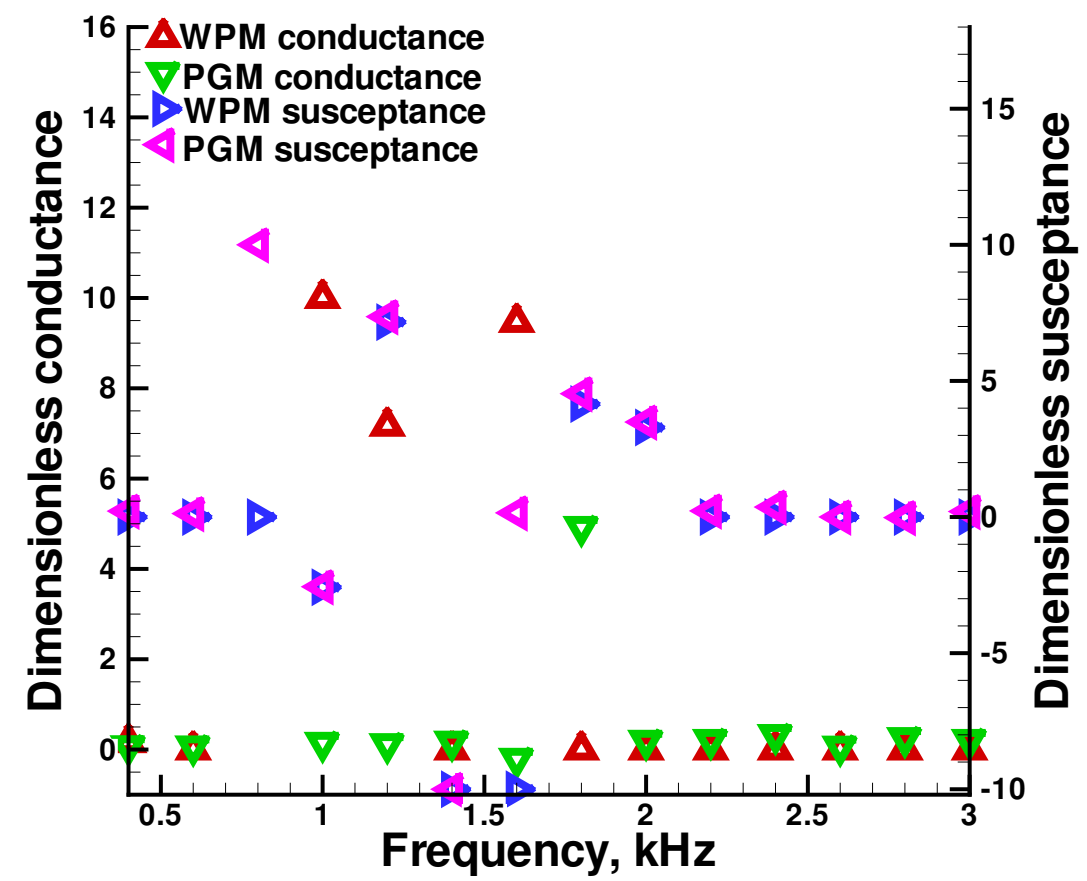

Figure 8. Educed dimensionless admittance of hardwall insert at Mach 0.5 (measured eduction data).

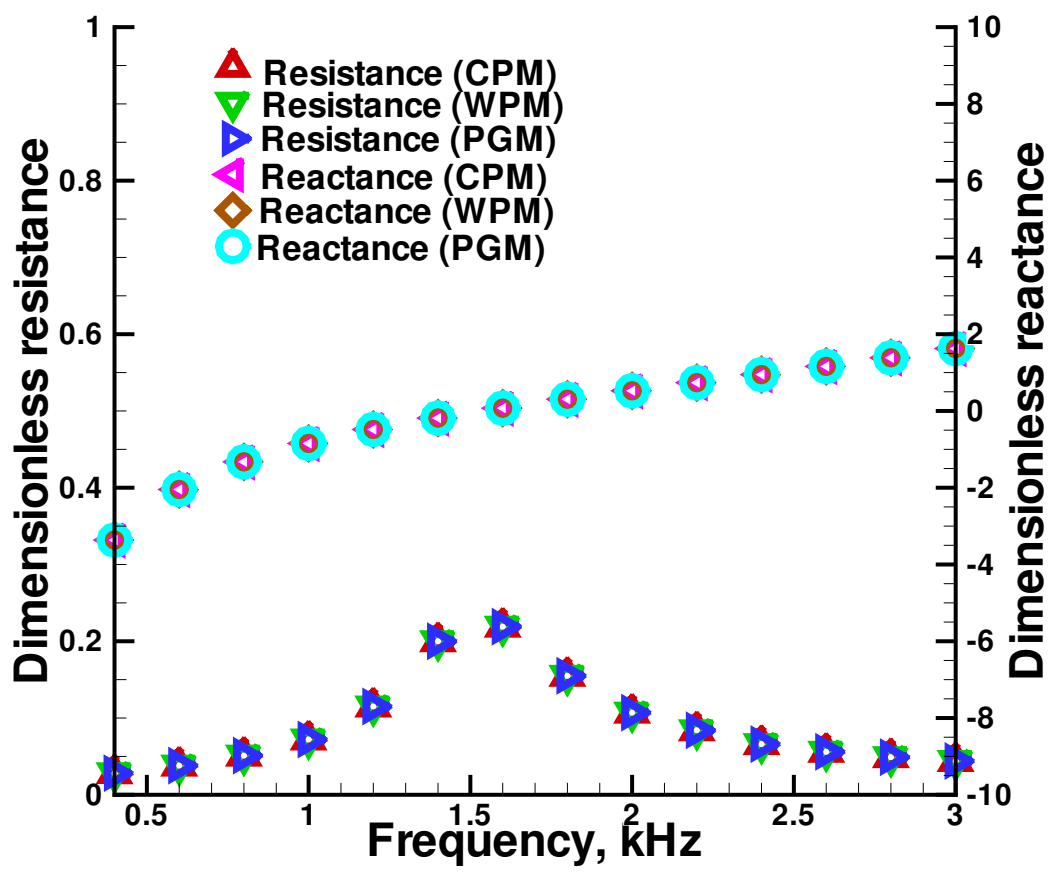

Figure 9. Educed dimensionless impedance of conventional test liner at Mach 0.0 (synthesized data). 


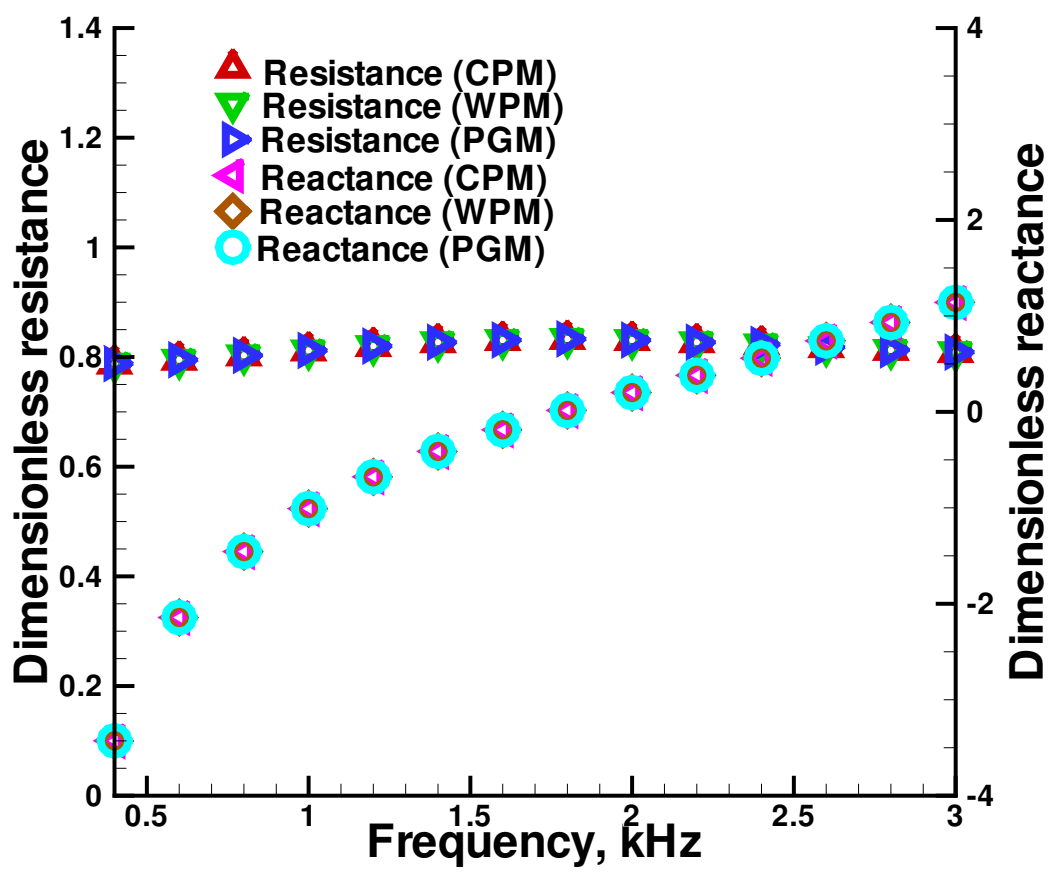

Figure 10. Educed dimensionless impedance of conventional test liner at Mach 0.3 (synthesized data).

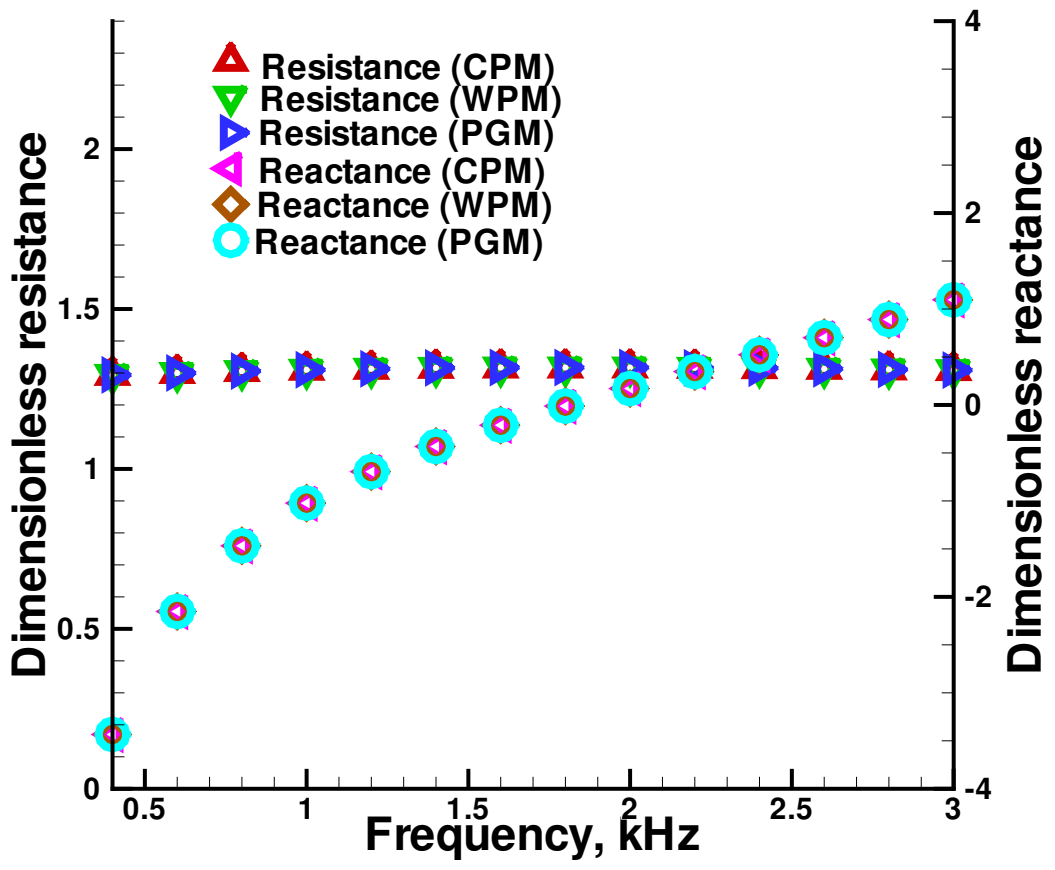

Figure 11. Educed dimensionless impedance of conventional test liner at Mach 0.5 (synthesized data). 


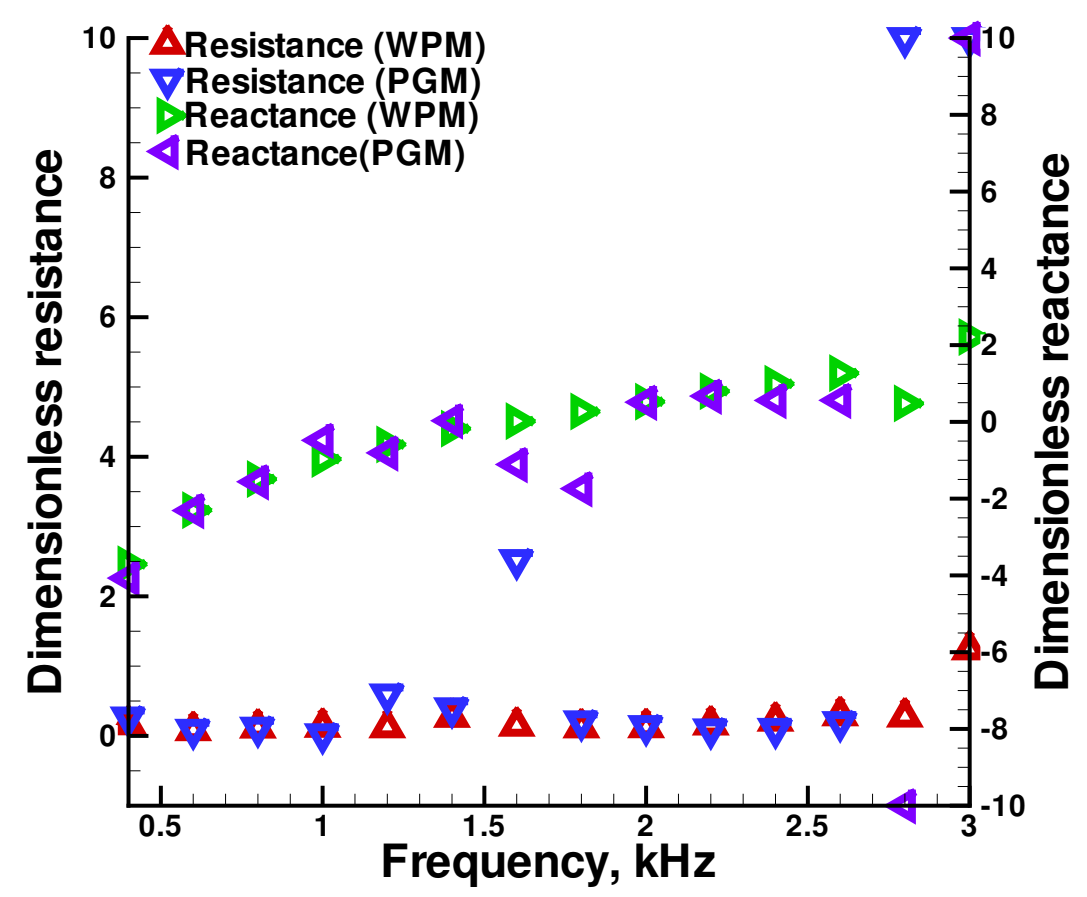

Figure 12. Educed dimensionless impedance of conventional liner at Mach 0.0 (measured data).

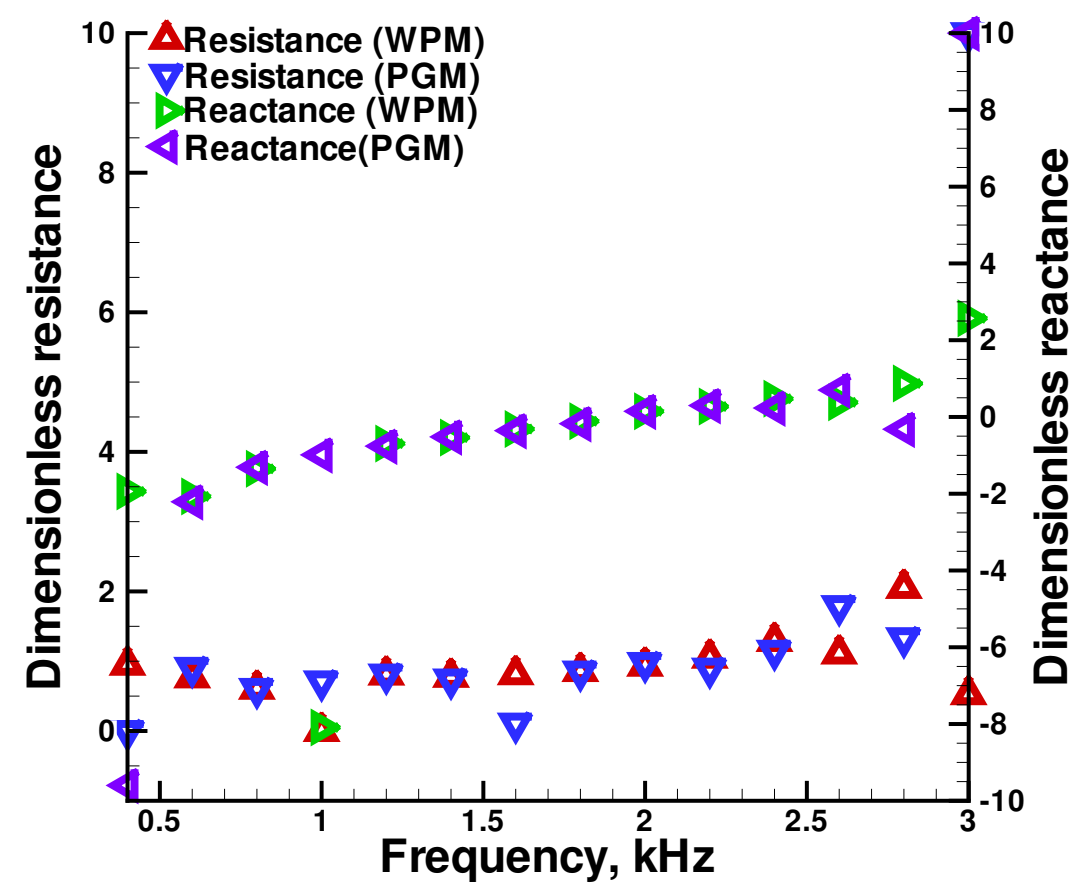

Figure 13. Educed dimensionless impedance of conventional liner at Mach 0.3 (measured data). 


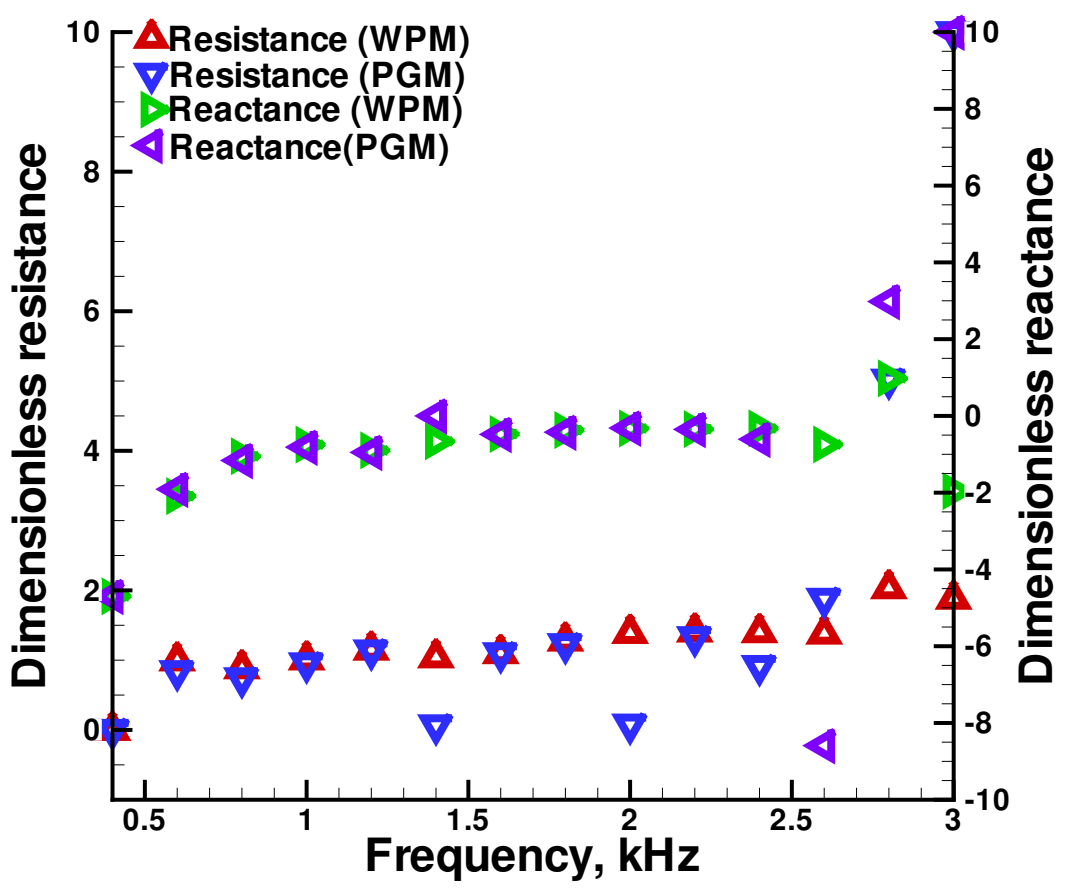

Figure 14. Educed dimensionless impedance of conventional liner at Mach 0.5 (measured data). 